Original Research Paper

\title{
Binding Interaction between Bovine Serum Albumin and Chicoric Acid, a Food Functional Component
}

\author{
${ }^{1}$ Haifang Xiao, ${ }^{2}$ Quancai Sun, ${ }^{2}$ Xuebo Liu and ${ }^{1}$ Yuanda Song \\ ${ }^{I}$ Colin Ratledge Center for Microbial Lipids, School of Agricultural Engineering and Food Science, \\ Shandong University of Technology, Zibo, PR China \\ ${ }^{2}$ College of Food Science and Engineering, Northwest A\&F University, Yangling, PR China
}

\author{
Article history \\ Received: 26-06-2016 \\ Revised: 18-07-2016 \\ Accepted: 15-09-2016 \\ Corresponding Author: \\ Haifang Xiao and Yuanda Song \\ Colin Ratledge Center for \\ Microbial Lipids, School of \\ Agricultural Engineering and \\ Food Science, Shandong \\ University of Technology, \\ Zibo, PR China \\ Email: xiaohaifang@sdut.edu.cn; \\ songyuanda@sdut.edu.cn
}

\begin{abstract}
Fuorescence, FTIR and UV-Vis absorption spectroscopy were used to explore the binding between chicoric acid and Bovine Serum Albumin (BSA). Binding characteristics at various levels of temperature have been calculated. The results indicated that chicoric acid statically quenched the intrinsic fluorescence of BSA. The binding constants $\left(K_{a}\right)$ were $4.14 \times 10^{5} \mathrm{~L} \mathrm{~mol}^{-1}$ at $273 \mathrm{~K}$ and $4.29 \times 10^{6} \mathrm{~L} \mathrm{~mol}^{-1}$ at $298 \mathrm{k}$. The numbers of binding sites between chicoric acid and BSA were both approximately equal to 1 at the two temperatures. Furthermore, the binding distance between chicoric acid and BSA was $2.69 \mathrm{~nm}$ which was calculated according to the Förster's resonance energy transfer. Thermodynamic parameters suggested that BSA bind chicoric acid spontaneously mainly via hydrophobic interaction. Results demonstrated that the conformation and microenvironment of BSA were changed after binding with chicoric acid. Moreover, chicoric acid showed stronger binding with tryptophan (Trp) residue than with tyrosine (Tyr) residue. Our results can provide scientific basis for studying availability and distribution of chicoric acid.
\end{abstract}

Keywords: Chicoric Acid Bovine Serum Albumin, Spectroscopy, Interaction, Conformation

\section{Introduction}

Phenolic acids widely occur in plant leaves, roots and especially fruits, are aromatic acid compounds and secondary plant metabolites (Herrmann and Nagel, 1989). Hydroxybenzoic and hydroxycinnamic acids are two groups of phenolic acids that widely distribute in plants (Ghasemzadeh and Ghasemzadeh, 2011). Much attention has been paid to these natural phenolic acids because of their functional activities in intervening diabetes, inflammatory and cancer as well as antioxidative and anti-microbial properties (Chao et al., 2009; Cueva et al., 2010; Hsu et al., 2000; Maurya et al., 2010; Nayaka et al., 2010). Moreover, previous reports revealed that the binding between some phenolic acids and biomolecules such as DNA and proteins played a certain role in their biological properties (Labieniec and Gabryelak, 2005).

Chicoric acid (Fig. 1) is a member of phenolic acids and found in many edible plants such as Echinacea purpurea, dandelion, basil, iceberg lettuce, chicory, cat's Whisker (Baur et al., 2004; Innocenti et al., 2005; Lee and Scagel, 2009; Liu et al., 2006; Olah et al., 2003;
Schütz et al., 2005). Many literatures have reported that chicoric acid possesses anti-oxidative, antivirus and anti-diabetic activities (Dalby-Brown et al., 2005; Robinson et al., 1996; Tousch et al., 2008). Moreover, previous study also revealed that chicoric acid possessed a stimulatory effect on phagocytes (Bone, 1997). Recently, the binding study of chicoric acid with HIV-1 integrase has been carried out (Healy et al., 2009).

Binding studies of small molecules to proteins are very important in their disposition and efficacy because protein binding can influence the effective solubility, distribution and biological half-life of small molecules in vivo as well as interaction between small molecules and other endogenous or exogenous compounds. Therefore, it is of great necessity for explaining the pharmacodynamics and pharmacokinetics of small molecules to investigate the binding between them and proteins (Cui et al., 2008; Qin et al., 2007). Serum albumins, lipoproteins and alglycoprotein are proteins commonly participated in protein binding (Abdi et al., 2012). Among them, the most abundant blood proteins are serum albumins which play an important role in balancing the oncotic pressure and pH of blood (Carter and Ho, 1994). 


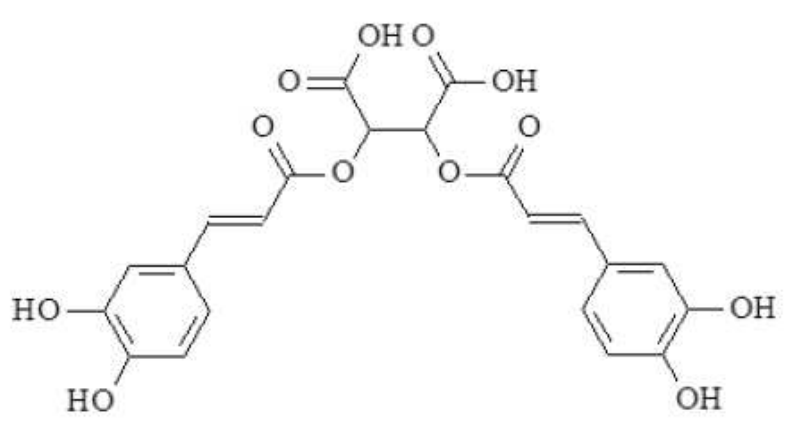

Fig. 1. Molecule structure of chivoric acid

The most prominent characteristic of serum albumins is that they can act as the depot proteins and transporters for numerous endogenous and exogenous small molecules (Huang et al., 2004). BSA was used frequently in previous studies because of its advantages such as highly stability, cheap and homology with Human Serum Albumin (HSA) in structure (Carter et al., 1994; Naik et al., 2010). BSA contains two tryptophan (Trp) residues including Trp-134 and Trp-212 which possess intrinsic fluorescence. In the first domain Trp134 is located on the surface of the molecule and in the second domain Trp-212 is located within a hydrophobic binding pocket of the protein (He and Carter, 1992; Hamdanim et al., 2009). The conformation of BSA would be changed upon interacting with small molecules.

UV-Vis absorption and fluorescence spectroscopy were used to investigate the interactions between serum albumins and small molecules for their outstanding sensitivity, selectivity, reproducibility, convenience and theoretical foundation (Zhang et al., 2012). FTIR spectroscopy is reliable method to illustrate the conformational changes of proteins after binding with small molecules (Darwish et al., 2010). Recently, several researches on the binding between phenolic acid and serum albumins have been undertaken using spectroscopic technology (Kang et al., 2004; Labieniec and Gabryelak, 2006; Meng et al., 2012; Rawel et al., 2005; Soares et al., 2007). However, no report of chicoric acid-serum albumins interaction has been found so far. Therefore, this research was carried out to explore the interaction between chicoric acid and BSA under simulated physiological conditions using fuorescence, FTIR and UV-Vis absorption spectroscopy. The mechanism of interaction between chicoric acid and BSA including quenching mechanism, binding parameters, binding distance, thermodynamic parameters and conformational change were explored.

\section{Materials and Methods}

\section{Chemicals and Reagents}

Chicoric Acid and BSA was obtained from Sigma Chemical Co. (St. Louis, MO, USA). All other reagents used in this study were of analytical purity. Water used throughout the experiments was ultrapure.

\section{Instrumentations}

Hitachi F-4500s pectrofluorimeter (Tokyo, Japan) with a $1.0 \mathrm{~cm}$ quartz cell and a $150 \mathrm{~W}$ xenon lamp was employed in this study to record fluorescence spectra. Excitation wavelength was set at $285 \mathrm{~nm}$. The widths of excitation slit and emission slit were both $10 \mathrm{~nm}$. The UV-Vis absorption spectra were measured by Shimadzu UV-2550 s pectrophotometer (Kyoto, Japan) in the wavelength range $250-350 \mathrm{~nm}$. FTIR spectra were measured using Thermo-Nicolet Avatar330 FTIR spectrometer (Rochester, NY, USA) using KBr pellets. The weight of samples was measured by Sartorius BP211D analytical balance with a precision of $0.1 \mathrm{mg}$ (Göttingen, Germany). PHS-3Cdigital pHmeter (Shanghai, China) was used to detect $\mathrm{pH}$ values.

\section{Preparation of Stock Solutions}

To keep the ionic strength of solution $\mathrm{NaCl}(0.10 \mathrm{M})$ was used in Tris- $\mathrm{HCl}$ buffer (0.10M, $\mathrm{pH} 7.4)$. All BSA solutions were prepared in Tris-HCl buffer solution and kept at $0-4{ }^{\circ} \mathrm{C}$ before used. The stock solution of chicoric acid was prepared in methanol.

\section{Fluorescence Studies}

Equal volumes of chicoric acid solutions with various concentrations were added to protein solutions, respectively. All solutions were mixed thoroughly. The final concentrations of chicoric acid were $0,2,3,4,5,6,7$, 8,9 and $10 \mu \mathrm{M}$. Then the mixtures of chicoric acid and BSA were equilibrated at 273 or $298 \mathrm{~K}$ for $20 \mathrm{~min}$. The fluorescence emissions spectra were recorded in the range of 300-450 nm and the binding constants of chicoric acidBSA systems were calculated in the base of fluorescence data. The synchronous fluorescence spectra of BSA with or without chicoric acid were recorded with the excitation and emission wavelength intervals $(\Delta \lambda)$ at 15 and $60 \mathrm{~nm}$, respectively. All the experiments were carried out in triplicate and the measurement error was less than $1 \%$.

\section{Absorption Studies}

The UV-Vis spectra were collected by Shimadzu UV2550 spectrophotometer in the region of $200-450 \mathrm{~nm}$ at $298 \mathrm{~K}$. The final concentrations of chicoric acid were 0,2 , 3,4 and $5 \mu \mathrm{M}$, respectively. While that of BSA was $1 \mu \mathrm{M}$.

\section{FTIR Spectroscopic Measurements}

The FTIR spectra of Tris- $\mathrm{HCl}$ buffer, BSA in the absence and presence of chicoric acid were collected in the spectral region $1000-2000 \mathrm{~cm}^{-1}$, respectively. Then the FTIR spectra of the sample solution were obtained by subtracting that of Tris-HCl buffer which taken as blank. 


\section{Results and Discussion}

\section{Binding Characteristics}

Molecular interaction is one of the causes to decrease the fluorescence intensity of a fluorophore (Vijayabharathi et al., 2012). Therefore, the interactions between small molecules and proteins were revealed through detecting fluorescence quenching. In this study, the fluorescence spectra of BSA $\left(\lambda_{\text {ex }}=285 \mathrm{~nm}\right)$ mixed with chicoric acid were obtained at 273 and $298 \mathrm{~K}$. Figure 2 shown that chicoric acid decreased the fluorescence intensity of BSA and there was a positive correction between concentrations of chicoric acid and fluorescence intensity of BSA. These results indicated that the interaction between chicoric acid and BSA occured and the non-fluorescent complex chicoric acid-BSA formed. Moreover, the emission maximum ( $\lambda \mathrm{em}$ ) of BSA slight red-shifted in the present of chicoric acid, indicating that Trp chromophore in BSA was located in a more hydrophilic environment because of the interaction of chicoric acid with BSA. This result was further confirmed by synchronous fluorescence spectra described below.

Dynamic and static quenching are two main mechanisms of fluorescence quenching and different in dependence on temperature and viscosity. Stern-Volmer equation (Lakowicz and Weber, 1973) (Equation 1) was usually used to analyze the quenching mechanism in the previous studies:

$$
F_{0} / F=1+K_{s V}[Q]=1+K_{q} \tau_{0}[Q]
$$

Where:

$F_{0}$ and $F=$ The fluorescence emission intensities with and without quencher, respectively

$K_{s v} \quad=$ The Stern-Volmerquenching constant

$K_{q} \quad=$ The quenching rate constant

$[Q] \quad=$ The concentration of quencher

$\tau_{0} \quad=$ The average lifetime of the molecules without quencher and its value is about $10^{-8} \mathrm{~S}$

Figure 3 showed the Stern-Volmer plots for BSA fluorescence quenched bychicoric acid. Satisfactory linearity of the Stern-Volmer equations was obtained in the investigated concentrations of chicoric acid. Table 1 listed the values of $K_{s v}$ and $K_{q}$. The results suggested that with temperatures rising the values of $K_{s v}$ decreased, indicating that static quenching was the probable machenism of fluorescence quenching between chicoric acid and BSA. Moreover, the quenching rate constants $\left(K_{q}\right)$ of BSA were determined to be $9.835 \times 1012$ and $7.454 \times 1012 \mathrm{~L} \mathrm{~mol}^{-1} \mathrm{~s}^{-1}$, respectively, which were far greater than the maximum diffusion collision quenching rate constant $\left(2.0 \times 10^{10} \mathrm{~mol}^{-1} \mathrm{Ls}^{-1}\right)$, further demonstrating that the dominant mechanism was static quenching in the fluorescence quenching process of BSA by chicoric acid.

\section{Binding Constants and Binding Sites}

The double-logarithm equation (Bandyopadhyay et al., 2012) (Equation 2) was used to caculate the binding constant $\left(K_{a}\right)$ and the number of binding sites $(n)$ in static quenching interaction:

$$
\lg \left[\frac{F_{0}-F}{F}\right]=\lg K_{a}+n \lg [Q]
$$

Figure 4 demonstrated plots of $\lg \left(F_{0^{-}} F\right) / F$ versus $\lg [Q]$ for chicoric acid-BSA. The values of $K_{a}$ and $n$ can be obtained from the intercept and the slope, respectively. The calculated $K_{a}$ and $n$ at different levels of temperature were summarized in Table 2. The values of $n$ at 273 and $298 \mathrm{~K}$ were both equal to 1, suggesting single class of binding site in BSA for chicoric acid.

\section{Thermodynamic Parameters and Binding Force}

Generally, the non-covalent interaction of small molecules and proteins cover hydrogen-bonding forces, vander Waals forces, hydrophobic interactions and electrostatic interactions. The major evidences for determining the binding mode of small molecule-protein are thermodynamic parameters such as free energy change $(\Delta \mathrm{G})$, enthalpy change $(\Delta \mathrm{H})$ and entropy change $(\Delta \mathrm{S})$. The parameters above can be estimated from Equation 3 and 4 :

$\operatorname{In} \frac{K_{a 2}}{K_{a 1}}=\frac{1}{R}\left[\frac{1}{T_{1}}-\frac{1}{T_{2}}\right] \Delta H$

$\Delta G=\Delta H-T \Delta S=-R T \ln K_{a}$

Where:

$K_{a 1}$ and $K_{a 2}=$ Binding constants at temperature $T_{1}$ and $T_{2}$, respectively

$R$ $=$ The gas constant

The interaction researches were implymented at 273 and $298 \mathrm{~K}$. Table 3 listed the thermodynamic parameters for the binding of chicoric acid and BSA. The value of $\Delta \mathrm{G}$ was negative indicating the binding process of chicoric acid and BSA was spontaneous. The values of $\Delta \mathrm{H}$ and $\Delta \mathrm{S}$ were positive implied that the interaction between chicoric acid and BSA was mainly an endothermic and entropy-driven reaction. Meanwhile, the main force between chicoric acid and BSA was hydrophobic force (Zhang et al., 2012).

\section{Binding Distance}

The spectral researches revealed that a complex was formed between chicoric acid and BSA. Additionally, Fig. 5 showed the fluorescence emission spectrum of BSA and the absorption spectrum of chicoric acid. 


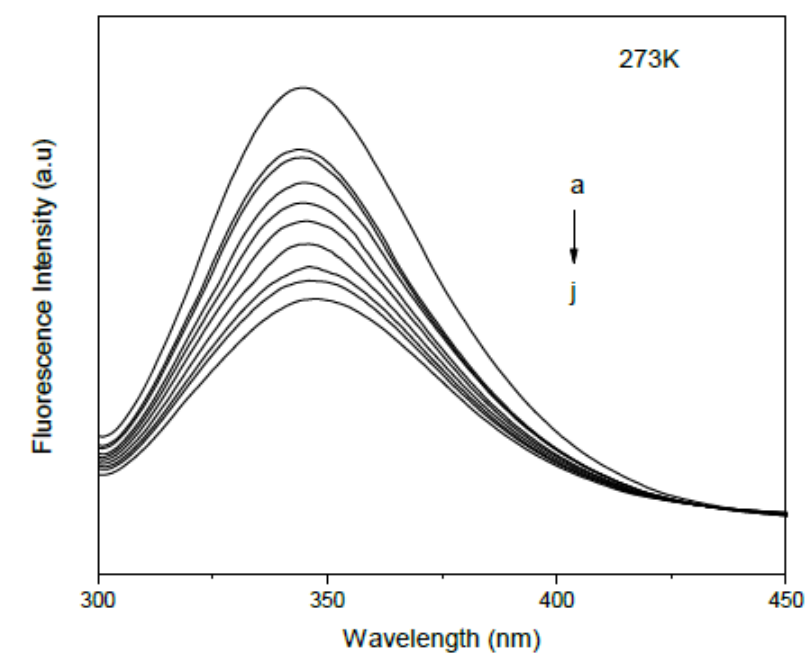

(a)

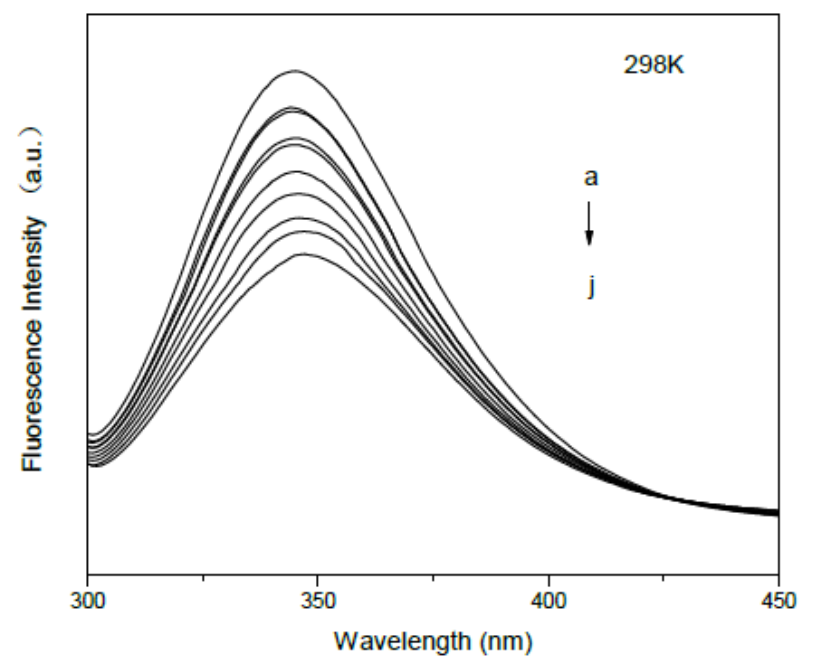

(b)

Fig. 2. The fluorescence spectra of BSA quenched by chicoric acid at $273 \mathrm{~K}$ (A) and $298 \mathrm{~K}$ (B). $\lambda_{\mathrm{ex}}=285 \mathrm{~nm}$; BSA, $1 \mu \mathrm{M}$; chicoric acid (a-j): $0,2,3,4,5,6,7,8,9$ and $10 \mu \mathrm{M}$, respectively

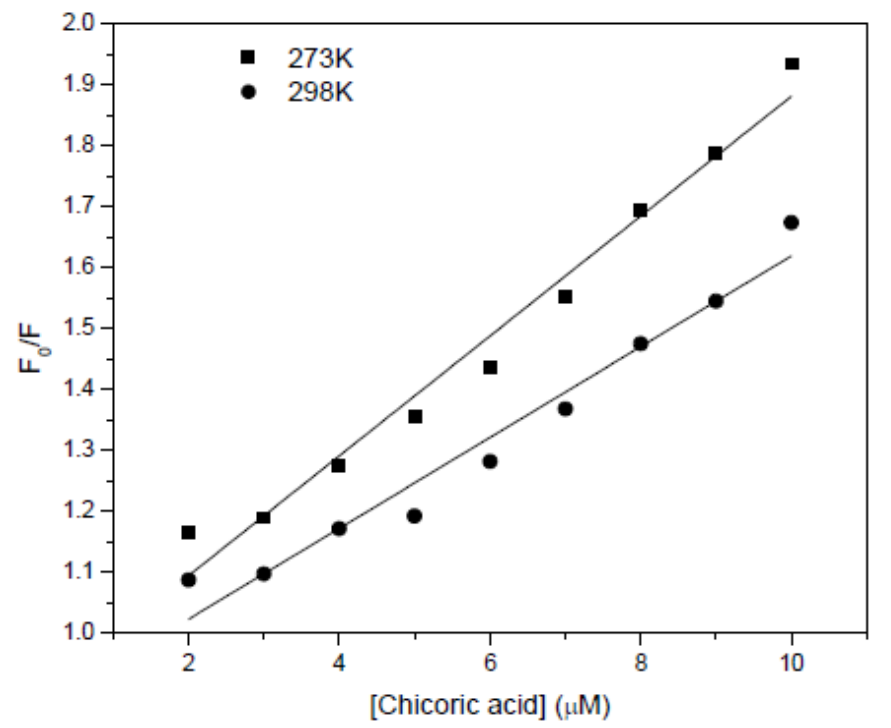

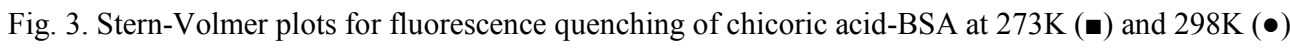

An overlap of the two spectra could be found. Therefore, the binding distance of chicoric acid and BSA can be obtained upon fluorescence resonance energy transfer. Equation 5 (Pang et al., 2012) could be used to determine the efficiency of energy transfer between the donor and acceptor $(\mathrm{E})$ :

$$
E=1-\frac{F}{F_{0}}=\frac{R_{0}^{6}}{R_{0}^{6}+r^{6}}
$$

Where:

$F$ and $F_{0}=$ The fluorescence intensities of BSA with and without chicoric acid, respectively $r \quad=$ The binding distance of BSA and chicoric acid $50 \%$ of the excitation energy is shifted to the acceptor at the critical distance $\left(R_{0}\right)$ which can be determined by the Equation 6 (Pang et al., 2012):

$R_{0}^{6}=8.8 \times 10^{-25} K^{2} n^{-4} \varnothing J$

Where:

$K_{2}=$ The spatial orientation factor of the dipole

$n=$ The refractive index of the medium

$\varphi=$ fluorescence quantum yield of the donor 


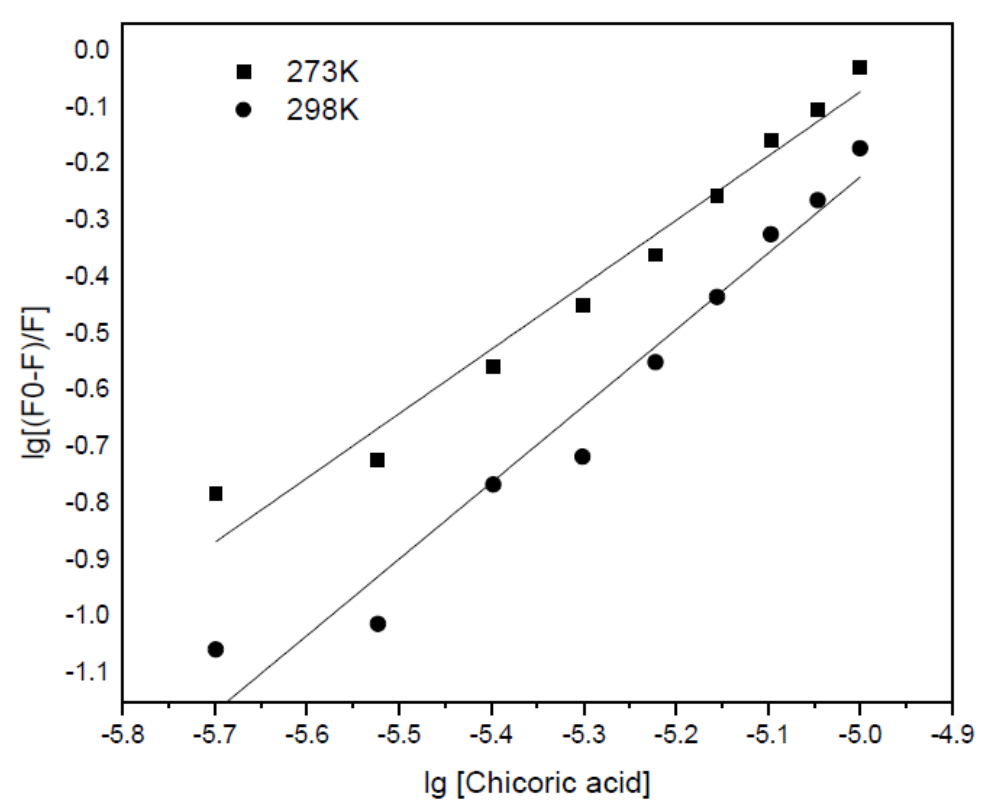

Fig. 4. Double log plots of BSA with chicoric acid at 273K (•) and 298K (•)

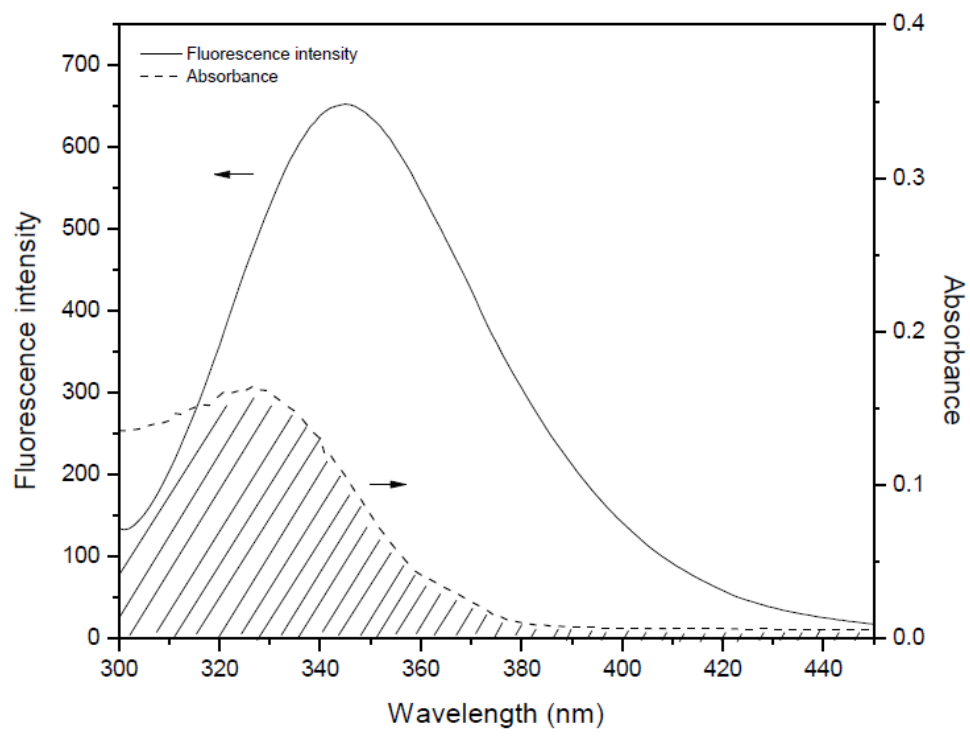

Fig. 5. The overlap of the fluorescence spectrum of BSA and the absorption spectrum of chicoric acid. BSA, $1 \mu \mathrm{M}$; chicoric acid, $10 \mu \mathrm{M} ; \mathrm{T}=298 \mathrm{~K}$

Table 1. Stern-Volmer quenching constants for the interaction of chicoric acid with BSA

\begin{tabular}{llll}
\hline $\begin{array}{l}\text { Temperature } \\
(\mathrm{K})\end{array}$ & $\begin{array}{l}K_{s v} \\
\left(\times 10^{4} \mathrm{Lmol}^{-1}\right)\end{array}$ & $\begin{array}{l}K_{q} \\
\left(\times 10^{12} \mathrm{~L} \mathrm{~mol}^{-1} \mathrm{~s}^{-1}\right)\end{array}$ & $\mathrm{r}$ \\
\hline $273 \mathrm{~K}$ & 9.835 & 9.835 & 0.9749 \\
$298 \mathrm{~K}$ & 7.454 & 7.454 & 0.9589 \\
\hline
\end{tabular}

Table 2. Binding constants and binding sites for the interaction of chicoric acid with BSA

\begin{tabular}{llll}
\hline $\begin{array}{l}\text { Temperature } \\
(\mathrm{K})\end{array}$ & $\begin{array}{l}K_{q} \\
\left(\mathrm{~L} \mathrm{~mol}^{-1}\right)\end{array}$ & $n$ & $r$ \\
\hline $273 \mathrm{~K}$ & 9.835 & 9.835 & 0.9749 \\
$298 \mathrm{~K}$ & 7.454 & 7.454 & 0.9589 \\
\hline
\end{tabular}

Table 3. Thermodynamic parameters for the binding of chicoric acid and BSA

\begin{tabular}{llll}
\hline $\begin{array}{l}\text { Temperature } \\
(\mathrm{K})\end{array}$ & $\begin{array}{l}\Delta \mathrm{G} \\
\left(\mathrm{kJ} \cdot \mathrm{mol}^{-1}\right)\end{array}$ & $\begin{array}{l}\Delta \mathrm{S} \\
\left(\mathrm{J} \cdot \mathrm{mol}-1 \cdot \mathrm{K}^{-1}\right)\end{array}$ & $\begin{array}{l}\Delta \mathrm{H} \\
\left(\mathrm{kJ} \cdot \mathrm{mol}^{-1}\right)\end{array}$ \\
\hline $273 \mathrm{~K}$ & -12.75 & 147.33 & 27.47 \\
$298 \mathrm{~K}$ & -16.43 & & \\
\hline
\end{tabular}

The values of $K^{2}, \varphi$ and $\mathrm{n}$ have been reported for BSA are 2/3, 0.14 and 1.36, respectively (Zhuang et al., 2012). J, the overlap integral of the fluorescence emission spectrum of donor and absorption spectrum of the acceptor, can be obtained using Equation 7 (Pang et al., 2012): 


$$
J=\frac{\Sigma F(\lambda) \varepsilon(\lambda) \lambda^{4} \Delta \lambda}{\Sigma F(\lambda) \Delta \lambda}
$$

Where:

$F(\lambda)=$ The fluorescence intensity of fluorescent donor at wavelength $\lambda$

$\varepsilon(\lambda)=$ The molar absorption coefficient of the acceptor at wavelength $\lambda$

Basing on Equation 5, 6 and 7, it could be obtained that $J=1.07 \times 10^{-14} \mathrm{~cm}^{3} \cdot \mathrm{L} \cdot \mathrm{mol}^{-1}, R_{0}=2.52 \mathrm{~nm}, E=0.40$, $r=2.69 \mathrm{~nm}$. The value of $r$ is less than $8 \mathrm{~nm}$, suggesting the shift of non-radiative energy between BSA and chicoric acid. Moreover, the value of $r$ was larger than that of $R_{0}$ also suggested that the quenching mechanism of chicoric acid to BSA was static (Zhuang et al., 2012). Base on the above, the static quenching combined with non-radiative energy transfer was the quenching mechanism for chicoric acid to BSA.

\section{Conformation Investigation}

\section{Synchronous Fuorescence Spectra}

Synchronous fluorescence spectroscopy can be used to investigate the changes in structure and conformation of proteins. The shift in maximum emission wavelength corresponds to changes in polarity around the chromosphere molecules (Jayabharathi et al., 2012). Synchronous fluorescence spectra offer information about the characteristics of Tyrresidue and Trpresidue when $\Delta \lambda$ between excitation wavelength and emission wavelength is fixed at 15 and $60 \mathrm{~nm}$, respectively (Shi et al., 2012). Figure 6 showed that with the increasing concentration of chicoric acid the fluorescence intensities at $\Delta \lambda=15$ and $\Delta \lambda=60 \mathrm{~nm}$ were both decreased gradually. The emission maximum of Tyr residue kept unchanged at $288 \mathrm{~nm}$ upon addition of

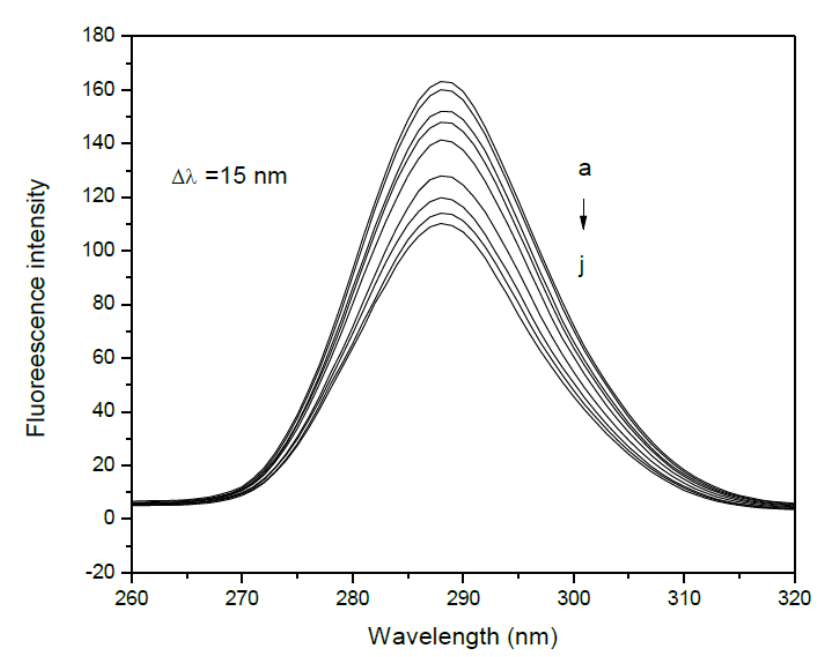

(a) chicoric acid, suggesting that chicoric acid had no obvious change on the microenvironment of the Tyr residue. Whereas, it was observed that the emission maximum of Trp residue had a weak red shift by about $1 \mathrm{~nm}$ from 283 $\mathrm{nm}$ to $284 \mathrm{~nm}$, indicating that Trp residue was close to chicoric acid, the hydrophobicity around the Trp residue decreased, however, the polarity increased (Zhuang et al., 2012). Additionally, we calculated fluorescence quenching ratios $\left(R_{S F Q}\right)$ basing on the equation $R_{S F Q}=1-F / F_{0}$. In this equation, $F$ and $F_{0}$ are the synchronous fluorescence intensities of BSA with and without chicoric acid, respectively (Meng et al., 2012). As shown in Fig. 7, the $R_{S F Q}$ for $\Delta \lambda=15 \mathrm{~nm}$ were smaller than the $R_{S F Q}$ for $\Delta \lambda=60$ $\mathrm{nm}$, suggesting that chicoric acid was more accessible to Trp residue than to Tyr residue (Wang et al., 2012).

\section{FTIR Measurements}

Different amide bands in infrared spectra of proteins indicate different vibrations of the peptide moiety. Two amide bands related with the secondary structure of protein were the protein amide I band at $1600-1700 \mathrm{~cm}^{-1}$ (mainly $\mathrm{C}=\mathrm{O}$ stretching) and $\mathrm{II}$ band at 1500-1600 $\mathrm{cm}^{-1}$ (C-N stretching coupled with $\mathrm{N}-\mathrm{H}$ bending mode). In this study, the binding between chicoric acid and BSA was demonstrated using infrared spectroscopy to obtain more information on mechanism of this interaction and conformational changes of BSA. Figure 8 showed the FTIR spectra of BSA and chicoric acid-BSA complex. The peak position of amide I and II bands shifted from 1654 to 1649 and 1542 to $1552 \mathrm{~cm}^{-1}$, respectively. The changes of these peak positions indicated that in the protein structural subunits $\mathrm{C}=\mathrm{O}$ and $\mathrm{C}-\mathrm{N}$ groups were bound with chicoric acid and then rearranged the polypeptide carbonyl hydrogen bonding pattern, finally changed the secondary structure of BSA (Tantipolphan et al., 2007).

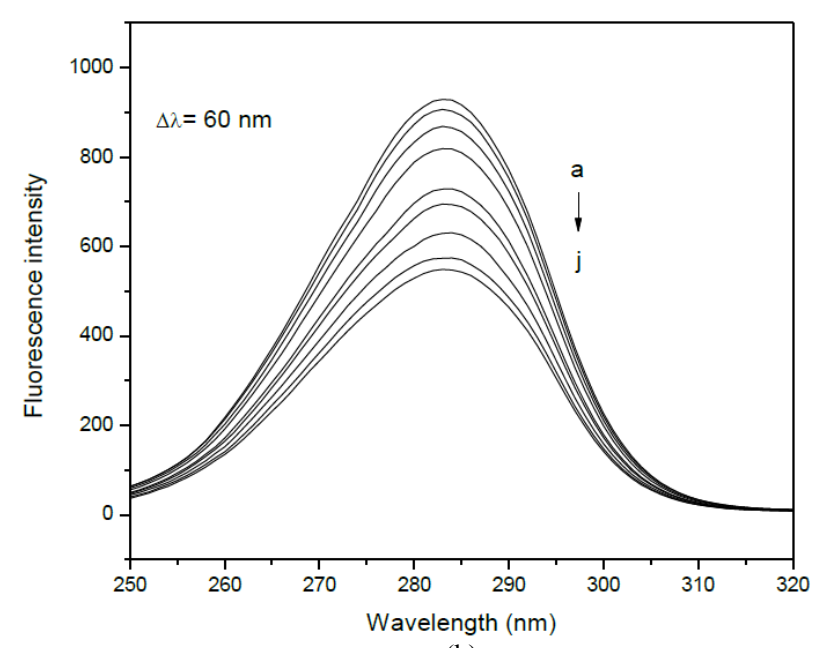

(b)

Fig. 6. The synchronous fluorescence spectra of BSA. (A) $\Delta \lambda=15 \mathrm{~nm}$, (B) $\Delta \lambda=60 \mathrm{~nm}$; BSA, $1 \mu \mathrm{M}$; chicoric acid (a-j): $0,2,3,4,5$, $6,7,8,9$ and $10 \mu \mathrm{M}$, respectively; $\mathrm{T}=298 \mathrm{~K}$ 


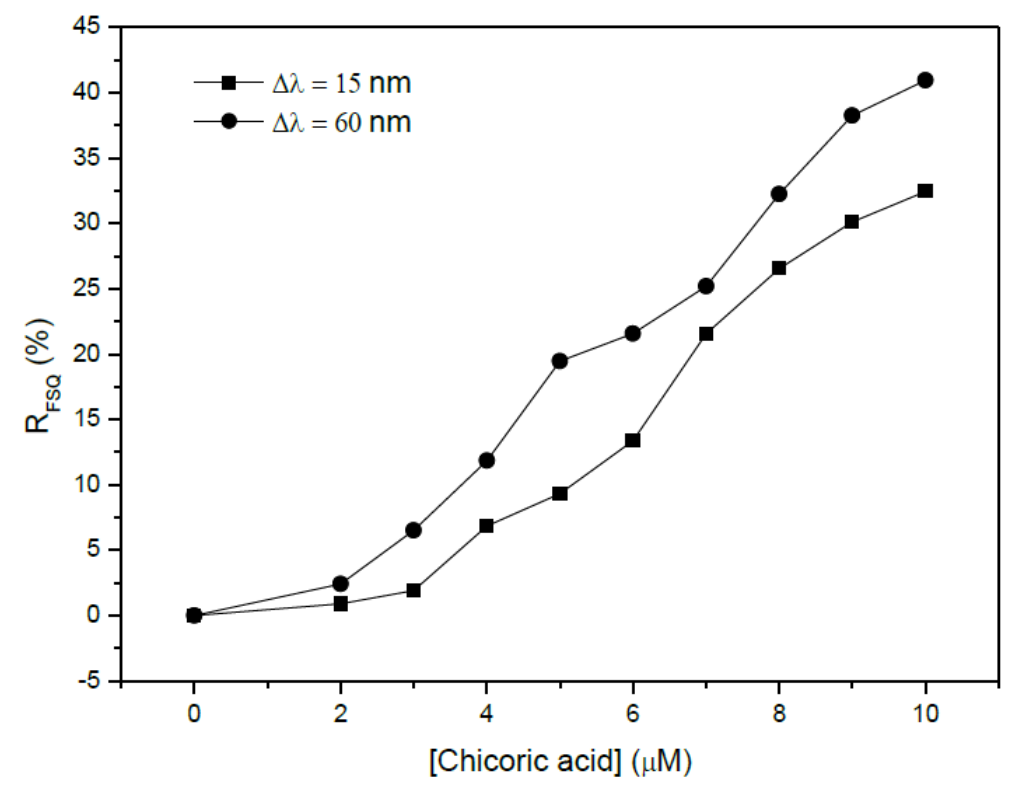

Fig. 7. Ratios of Synchronous Fluorescence Quenching (RSFQ) of BSA in the presence chicoric acid. BSA, $1 \mu \mathrm{M}$; chicoric acid: 0 , $2,3,4,5,6,7,8,9$ and $10 \mu \mathrm{M}$, respectively; $\mathrm{T}=298 \mathrm{~K} . \Delta \lambda=15 \mathrm{~nm}(\boldsymbol{\bullet}), \Delta \lambda=60 \mathrm{~nm}(\bullet)$

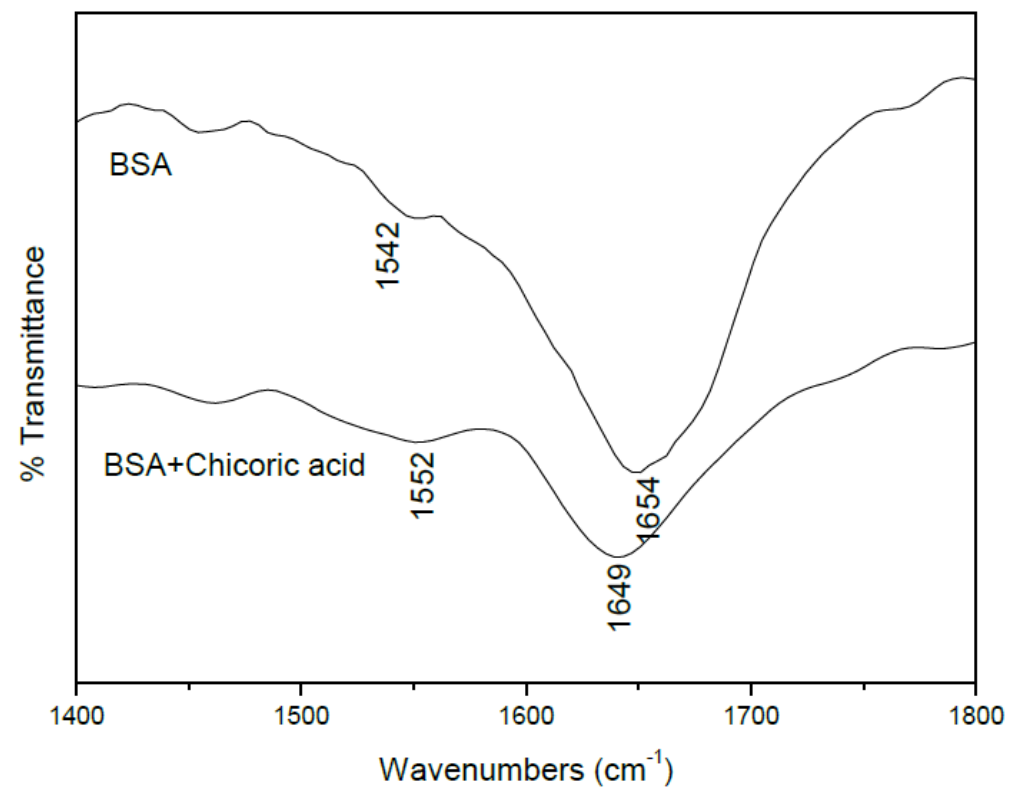

Fig. 8. FTIR spectrum of BSA and difference spectrum of BSA-chicoric acid complex. The FTIR spectrum of BSA was obtained by subtracting the spectrum of buffer solution from the spectrum of the protein solution; the FTIR difference spectrum of BSAchicoric acid complex was obtained by subtracting the spectrum of chicoric acid-free form from that of chicoric acid-BSA complex form; BSA, $1 \mu \mathrm{M}$; chicoric acid, $10 \mu \mathrm{M}$; T $=298 \mathrm{~K}$

\section{UV-Vis Absorption Studies}

For reconfirming the conformational change of BSA by the addition of chicoric acid, UV-Vis absorption spectra of BSA with varying concentrations of chicoric acid were obtained. In the present of chicoric acid, the absorption peak intensity of BSA increased as well as the peak red shifted from 278 to $286 \mathrm{~nm}$ (Fig. 9). It was reported that dynamic quenching did not change the absorption spectrum, but the formation of non-fluorescence ground-state complex can change it (Du et al., 2012). Thus, these results indicated that the interaction between chicoric acid and BSA caused the formation of ground sate complex and reconfirmed the static quenching mechanism of this interaction (Liu et al., 2010). 


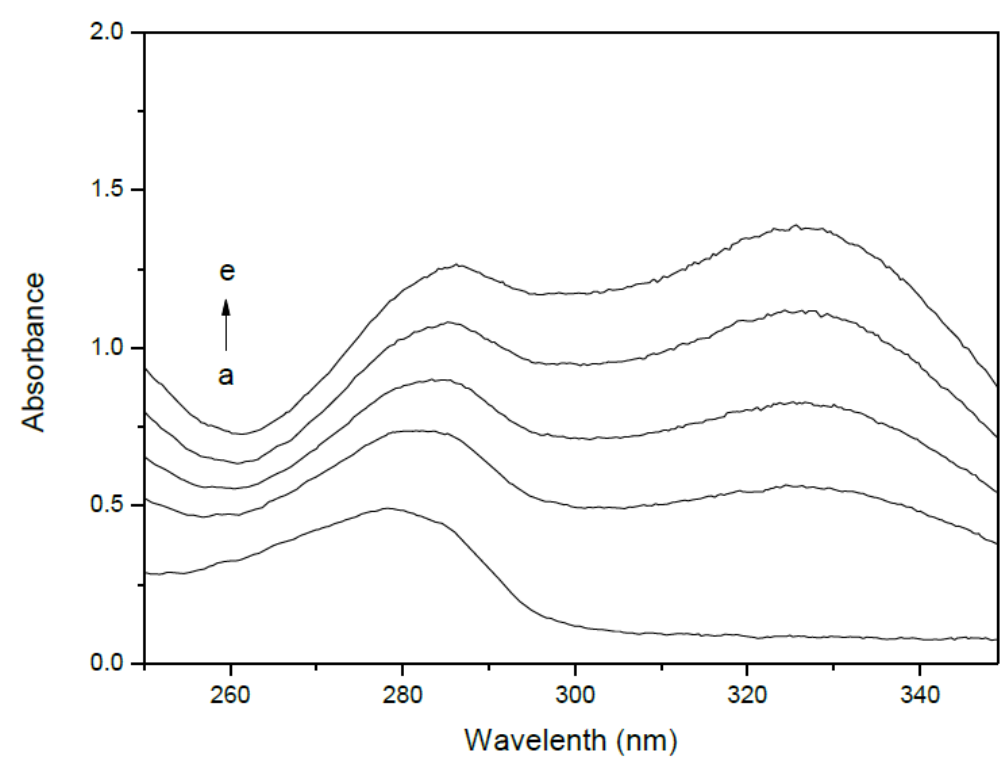

Fig. 9. UV-Vis absorption spectra of BSA and chicoric acid-BSA complex. BSA, $1 \mu \mathrm{M}$; chicoric acid, (a-e): 0, 2, 3, 4, $5 \mu \mathrm{M} ; \mathrm{T}=298 \mathrm{~K}$

\section{Conclusion}

In this study, the interaction of chicoric acid and BSA was studied using spectroscopic analysis. The results demonstrated that static quenching process was probable the quenching mechanism of BSA by chicoric acid. It was calculated that one binding site in BSA was accessible to chicoric acid. Thermodynamic parameters revealed that the binding reaction was spontaneous and hydrophobic force played a major role during the interaction. The distance between chicoric acid and BSA was less than 8 $\mathrm{nm}$ base on the Förster's resonance energy transfer. Additionally, BSA undergone conformational and microenvironment changes upon binding to chicoric acid and the binding site is mainly at Trp residue.

\section{Acknowledgement}

This work was supported by Shandong Provincial Natural Science Foundation, China (No. ZR2014CQ002). Therefore, we are grateful for this funding and support of this research.

\section{Funding Information}

This work was supported by Shandong Provincial Natural Science Foundation, China (No. ZR2014CQ002).

\section{Author's Contributions}

Haifang Xiao: Took part in all experiment process as well as data analysis and manuscript preparation.

Quancai Sun: Involved in study design and data analysis.
Xuebo Liu: Coordinated the study.

Yuanda Song: Integrated all data and improved the manuscript.

\section{Ethics}

This article is original and contains unpublished material. The corresponding author confirms that all of the other authors have read and approved the manuscript and no ethical issues involved.

\section{References}

Abdi, K., S. Nafisi, F. Manouchehri, M. Bonsaii and A. Khalaj, 2012. Interaction of 5-Fluorouracil and its derivatives with bovine serum albumin. J. Photochem. Photob. B: Biol., 107: 20-26. DOI: 10.1016/j.jphotobiol.2011.11.004

Bandyopadhyay, P., A.K. Ghosh and C. Ghosh, 2012. Recent developments on polyphenol-protein interactions: Effects on tea and coffee taste, antioxidant properties and the digestive system. Food Funct., 3: 592-605. DOI: 10.1039/C2FO00006G

Baur, S., R.G. Klaiber, A. Koblo and R. Carle, 2004. Effect of different washing procedures on phenolic metabolism of shredded, packaged iceberg lettuce during storage. J. Agr. Food Chem., 52: 7017-7025. DOI: $10.1021 / \mathrm{jf0} 48961 \mathrm{a}$

Bone, K., 1997. Echinacea: What makes it work. Alt. Med. Rev., 2: 87-93.

Carter, D.C., B. Chang, J.X. Ho, K. Keeling and Z. Krishnasami, 1994. Preliminary crystallographic studies of four crystal forms of serum albumin. Eur. J. Biochem., 226: 1049-1052.

DOI: $10.1111 /$ j.1432-1033.1994.01049.x 
Carter, D.C. and J.X. Ho, 1994. Structure of serum albumin. Adv. Protein Chem., 45: 153-203. DOI: $10.1016 / \mathrm{S} 0065-3233(08) 60640-3$

Chao, P.C., C.C. Hsu and M.C. Yin, 2009. Antiinflammatory and anti-coagulatory activities of caffeic acid and ellagic acid in cardiac tissue of diabetic mice. Nutr. Metab., 6: 1-8. DOI: $10.1186 / 1743-7075-6-33$

Cueva, C., M.V. Moreno-Arribas, P.J. Martín-Álvarez, G. Bills and M.F. Vicente et al., 2010. Antimicrobial activity of phenolic acids against commensal, probiotic and pathogenic bacteria. Res. Microbiol., 161: 372-382.

DOI: $10.1016 /$ j.resmic.2010.04.006

Cui, F., L. Qin, G. Zhang, Q. Liu and X. Yao et al., 2008. Interaction of anthracycline disaccharide with human serum albumin: Investigation by fluorescence spectroscopic technique and modeling studies. J. Pharmaceut. Biomed. Anal., 48: 1029-1036. DOI: 10.1016/j.jpba.2008.07.009

Dalby-Brown, L., H. Barsett, A.K.R. Landbo, A.S. Meyer and P. Mølgaard, 2005. Synergistic antioxidative effects of alkamides, caffeic acid derivatives and polysaccharide fractions from Echinacea purpureaon in vitro oxidation of human low-density lipoproteins. J. Agr. Food Chem., 53: 9413-9423. DOI: $10.1021 /$ jf0502395

Darwish, S.M., M.M.A. Teir, S.A. Makharza and M.M. Abu-Hadid, 2010. Spectroscopic investigations of pentobarbital interaction with human serum albumin. J. Mol. Struct., 963: 122-129. DOI: $10.1016 /$ j.molstruc.2009.10.023

Du, W., T. Teng, C.C. Zhou, L. Xi and J.Z. Wang, 2012. Spectroscopic studies on the interaction of bovine serum albumin with ginkgolic acid: Binding characteristics and structural analysis. J. Lumin., 132: 1207-1214. DOI: 10.1016/j.jlumin.2011.12.067

Ghasemzadeh, A. and N. Ghasemzadeh, 2011. Flavonoids and phenolic acids: Role and biochemical activity in plants and human. J. Med. Plants Res., 5: 6697-6703. DOI: 10.5897/JMPR11.1404

Hamdanim, S., D. Joly, R. Carpentier and H. TajmirRiahi, 2009. The effect of methylamine on the solution structures of human and bovine serum albumins. J. Mol. Struct., 936: 80-86. DOI: 10.1016/j.molstruc.2009.07.019

He, X.M. and D.C. Carter, 1992. Atomic structure and chemistry of human serum albumin. Nature, 358: 209-215. DOI: $10.1038 / 358209 a 0$

Healy, E.F., J. Sanders, P.J. King and W.E. Robinson, 2009. A docking study of L-chicoric acid with HIV1 integrase. J. Mol. Graph. Model., 27: 584-584. DOI: 10.1016/j.jmgm.2008.09.011

Herrmann, K. and C.W. Nagel, 1989. Occurrence and content of hydroxycinnamic and hydroxybenzoic acid compounds in foods. Crit. Rev. Food Sci., 28: 315-347. DOI: 10.1080/10408398909527504
Hsu, F.L., Y.C. Chen and J.T. Cheng, 2000. Caffeic acid as active principle from the fruit of Xanthium strumarium to lower plasma glucose in diabetic rats. Planta. Med., 66: 228-230. DOI: $10.1055 / \mathrm{s}-2000-8561$

Huang, B.X., H.Y. Kim and C. Dass, 2004. Probing three-dimensional structure of bovine serum albumin by chemical cross-linking and mass spectrometry. J. Am. Soc. Mass Spectrom., 15: 1237-1247. DOI: 10.1016/j.jasms.2004.05.004

Innocenti, M., S. Gallori, C. Giaccherini, F. Ieri and F.F. Vincieri et al., 2005. Evaluation of the phenolic content in the aerial parts of different varieties of Cichorium intybusL. J. Agr. Food Chem., 53: 6497-6502. DOI: 10.1021/jf050541d

Jayabharathi, J., V. Thanikachalam and M.V. Perumal, 2012. A study on the binding interaction between the imidazole derivative and bovine serum albumin by fluorescence spectroscopy. J. Lumin., 132: 707-712. DOI: 10.1016/j.jlumin.2011.10.023

Kang, J., Y. Liu, M.X. Xie, S. Li and M. Jiang et al., 2004. Interactions of human serum albumin with chlorogenic acid and ferulic acid. Biochim. Biophys. Acta (BBA)-Gen. Subjects, 1674: 205-214.

DOI: $10.1016 /$ j.bbagen.2004.06.021

Labieniec, M. and T. Gabryelak, 2005. Measurement of DNA damage and protein oxidation after the incubation of B14 Chinese hamster cells with chosen polyphenols. Toxicol. Lett., 155: 15-25. DOI: 10.1016/j.toxlet.2004.06.008

Labieniec, M. and T. Gabryelak, 2006. Interactions of tannic acid and its derivatives (ellagic and gallic acid) with calf thymus DNA and bovine serum albumin using spectroscopic method. J. Photochem. Photob. B: Biol., 82: 72-78.

DOI: 10.1016/j.jphotobiol.2005.09.005

Lakowicz, J.R. and G. Weber, 1973. Quenching of fluorescence by oxygen. Probe for structural fluctuations in macromolecules. Biochemistry, 12: 4161-4170. DOI: 10.1021/bi00745a020

Lee, J. and C.F. Scagel, 2009. Chicoric acid found in basil (Ocimum basilicumL.) leaves. Food Chem., 115: 650-656. DOI: 10.1016/j.foodchem.2008.12.075

Liu, B., C. Xue, J. Wang, C. Yang and F. Zhao et al., 2010. Study on the competitive reaction between bovine serum albumin and neomycin with ponceau S as fluorescence probe. J. Lumin., 130: 1999-2003. DOI: 10.1016/j.jlumin.2010.05.017

Liu, C.Z., B.H. Abbasi, M. Gao, S.J. Murch and P.K. Saxena, 2006. Caffeic acid derivatives production by hairy root cultures of Echinacea purpurea. J. Agr. Food Chem., 54: 8456-8460. DOI: 10.1021/jf061940r

Maurya, D.K., N. Nandakumar and T.P.A. Devasagayam, 2010. Anticancer property of gallic acid in A549, a human lung adenocarcinoma cell line and possible mechanisms. J. Clin. Biochem. Nutr., 48: 85-90. DOI: $10.3164 /$ jcbn.11-004FR 
Meng, F.Y., J.M. Zhu, A.R. Zhao, S.R. Yu and C.W. Lin, 2012. Synthesis of p-hydroxycinnamic acid derivatives and investigation of fluorescence binding with bovine serum albumin. J. Lumin., 132: 1290-1298. DOI: 10.1016/j.jlumin.2011.12.075

Naik, P., S. Chimatadar and S. Nandibewoor, 2010. Interaction between a potent corticosteroid drugdexamethasone with bovine serum albumin and human serum albumin: A fluorescence quenching and fourier transformation infrared spectroscopy study. J. Photochem. Photob. B: Biol., 100: 147-159. DOI: 10.1016/j.jphotobiol.2010.05.014

Nayaka, M.H., U. Sathisha and S.M. Dharmesh, 2010. Cytoprotective and antioxidant activity of free, conjugated and insoluble-bound phenolic acids from swallow root (Decalepis hamiltonii). Food Chem., 119: $1307-1312$.

DOI: 10.1016/j.foodchem.2009.08.044

Olah, N.K., L. Radu, C. Mogoşan, D. Hanganu and S. Gocan, 2003. Phytochemical and pharmacological studies on Orthosiphon stamineus Benth. (Lamiaceae) hydroalcoholic extracts. J. Pharmaceut. Biomed. Anal., 33: 117-123. DOI: 10.1016/S0731-7085(03)00227-9

Pang, B., S. Bi, Y. Wang, L. Yan and T. Wang, 2012. Investigation on the interactions of silymarin to bovine serum albumin and lysozyme by fluorescence and absorbance. J. Lumin., 132: 895-900. DOI: 10.1016/j.jlumin.2011.11.021

Qin, C., M.X. Xie and Y. Liu, 2007. Characterization of the myricetin-human serum albumin complex by spectroscopic and molecular modeling approaches. Biomacromolecules, 8: 2182-2189. DOI: $10.1021 / \mathrm{bm} 070319 \mathrm{c}$

Rawel, H.M., K. Meidtner and J. Kroll, 2005. Binding of selected phenolic compounds to proteins. J. Agr. Food Chem., 53: 4228-4235. DOI: $10.1021 / \mathrm{jf0} 480290$

Robinson, W.E., M.G. Reinecke, S. Abdel-Malek, Q. Jia and S.A. Chow, 1996. Inhibitors of HIV-1 replication that inhibit HIV integrase. P. Nati. Acad. Sci. USA., 93: 6326-6331. PMID: 8692814

Schütz, K., D.R. Kammerer, R. Carle and A. Schieber, 2005. Characterization of phenolic acids and flavonoids in dandelion (Taraxacum officinale WEB. ex WIGG.) root and herb by highperformance liquid chromatography/electrospray ionization mass spectrometry. Rapid Commun. Mass Sp., 19: 179-186. DOI: 10.1002/rcm.1767
Shi, Y., H. Liu, M. Xu, Z. Li and G. Xie et al., 2012. Spectroscopic studies on the interaction between an anticancer drug ampelopsin and bovine serum albumin. Spectrochim. Acta Part A: Mol. Biomol. Spectrosc., 87: 251-257. DOI: $10.1016 /$ j.saa.2011.11.048

Soares, S., N. Mateus and V. De Freitas, 2007. Interaction of different polyphenols with Bovine Serum Albumin (BSA) and Human Salivary $\alpha-$ amylase (HSA) by fluorescence quenching. J. Agr. Food Chem., 55: 6726-6735. DOI: $10.1021 / \mathrm{jf070905x}$

Tantipolphan, R., T. Rades, A. McQuillan and N. Medlicott, 2007. Adsorption of Bovine Serum Albumin (BSA) onto lecithin studied by attenuated total reflectance Fourier transform infrared (ATRFTIR) spectroscopy. Int. J. Pharm., 337: 40-47. DOI: $10.1016 /$ j.ijpharm.2006.12.021

Tousch, D., A.D. Lajoix, E. Hosy, J. Azay-Milhau and K. Ferrare et al., 2008. Chicoric acid, a new compound able to enhance insulin release and glucose uptake. Biochem. Bioph. Res. Co., 377: 131-135. DOI: 10.1016/j.bbrc.2008.09.088

Vijayabharathi, R., P. Sathyadevi, P. Krishnamoorthy, D. Senthilraja and P. Brunthadevi et al., 2012. Interaction studies of resistomycin from Streptomyces aurantiacus AAA5 with calf thymus DNA and bovine serum albumin. Spectrochim. Acta Part A: Mol. Biomol. Spectrosc., 89: 294-300. DOI: $10.1016 /$ j.saa.2011.12.072

Wang, Z., J. Li, J. Wang, M. Zou and S. Wang et al., 2012. Spectrometry researches on interaction and sonodynamic damage of Riboflavin (RF) to Bovine Serum Albumin (BSA). Spectrochim. Acta Part A: Mol. Biomol. Spectrosc., 87: 1-10. DOI: $10.1016 /$ j.saa.2011.11.006

Zhang, G., Y. Ma, L. Wang, Y. Zhang and J. Zhou, 2012. Multispectroscopic studies on the interaction of maltol, a food additive, with bovine serum albumin. Food Chem., 133: 264-270.

DOI: 10.1016/j.foodchem.2012.01.014

Zhuang, W., L. Li, G. Lin, Z. Deng and M. Peng, 2012. Ilaprazole metabolites, ilaprazole sulfone and ilaprazole sulfide decreased the affinity of ilaprazole to bovine serum albumin. J. Lumin., 132: 350-356. DOI: 10.1016/j.jlumin.2011.09.020 